\title{
New embeddings between the Higman-Thompson groups
}

\author{
J.C. Birget \\ 10.iii.2019
}

\begin{abstract}
We give a direct proof that all Higman-Thompson groups of the form $G_{k, 1}$ (for $k \geq 2$ ) are embedded in one another, which is a recent result of N. Matte Bon. This extends the embeddings given by Higman in 1974 .
\end{abstract}

\section{Introduction}

Higman [5, Theorem 7.2 and Lemma 7.1] showed that if $K=1+(k-1) d$ for some $d \geq 1$, then $G_{K, r} \leq G_{k, r}$. In particular, $G_{K, 1} \leq G_{2,1}$ for all $K \geq 2$. It is also known that all $G_{k, 1}$ are non-isomorphic for different $k$ ([5, Theorem 6.4], later generalized to the groups $G_{k, r}$ by [8]).

For a long time there has been a common belief that it was well known, and easy to prove, that all $G_{k, 1}$ embed into each other (see the comments in section 3). However, the first proof of this is quite recent; it follows from [6. Coroll. 11.16] near the end of a long paper by Nicolás Matte Bon.

Theorem 1.1 (N. Matte Bon). All Higman-Thompson groups $G_{k, 1}$ (for $k \geq 2$ ) are embedded in one another; i.e., for all $i, j \geq 2: G_{i, 1} \leq G_{j, 1}$.

The contribution of the present paper is a proof that is direct, elementary, and relatively short. In section 2 we prove that $G_{2,1} \leq G_{k, 1}$ for all $k>2$; this, in combination with Higman's embeddings, implies the Theorem.

The Higman-Thompson groups $G_{k, r}$ (for $k \geq 2, k>r \geq 1$ ) were introduced by Graham Higman [5] as a generalization of the Thompson group $V\left(=G_{2,1}\right)$ [10, 11]. We refer to the literature (in particular [10, 7, 11, 5, 9, 4]) for some of the remarkable properties of these groups; these groups occur in many subjects (e.g., Pardo used connections with Leavitt path algebras to prove his result [8]).

To define $G_{k, 1}$ we follow [1] (which is similar to [9] except for terminology). We use the alphabet $A_{k}=\left\{a_{0}, a_{1}, \ldots, a_{k-1}\right\}$, for any integer $k \geq 2$. Often we just write $A$ instead of $A_{k}$. The empty string is denoted by $\varepsilon$, and the set of all strings over $A$ is denoted by $A^{*}$, and the set of all nonempty strings is denoted by $A^{+}$. For a string $x \in A^{*},|x|$ denotes the length. For a set $S \subseteq A^{*},|S|$ denotes the cardinality. Concatenation of sets $S, T \subseteq A^{*}$ is denoted by $S T$ or $S \cdot T$, and defined by $S T=\{s t: s \in S, t \in T\}$. For $x, p \in A^{*}$ we say that $p$ is a prefix of $x$ iff $\left(\exists u \in A^{*}\right) x=p u$; this is denoted by $p \leq_{\operatorname{pref}} x$. Two strings $x, y \in A^{*}$ are called prefix-comparable (denoted by $x \|_{\text {pref }} y$ ) iff $x \leq_{\text {pref }} y$ or $y \leq$ pref $x$. A prefix code is any subset $P \subset A^{*}$ such that for all $p_{1}, p_{2} \in P: p_{1} \|_{\text {pref }} p_{2}$ implies $p_{1}=p_{2}$. A right ideal of $A^{*}$ is any subset $R \subseteq A^{*}$ such that $R=R \cdot A^{*}$. A subset $C \subseteq R$ generates $R$ as a right ideal iff $R=C \cdot A^{*}$. It is easy to prove that every finitely generated right ideal is generated by a unique finite prefix code, and this prefix code is the minimum generating set of the right ideal (with respect to $\subseteq$ ). A maximal prefix code is a prefix code $P \subset A^{*}$ that is not a strict subset of any other prefix code of $A^{*}$.

In this paper, function means partial function. For a function $f: A^{*} \rightarrow A^{*}$, the domain and image sets are denoted by $\operatorname{Dom}(f)$, respectively $\operatorname{Im}(f)$. A right ideal morphism of $A^{*}$ is a function $f: A^{*} \rightarrow A^{*}$ such that for all $x \in \operatorname{Dom}(f)$ and all $w \in A^{*}$ : 


$$
f(x w)=f(x) w .
$$

In that case, $\operatorname{Dom}(f)$ is a right ideal; one easily proves that $\operatorname{Im}(f)$ is also a right ideal. The prefix code that generates $\operatorname{Dom}(f)$ is denoted by $\operatorname{dom} C(f)$, and is called the domain code of $f$; the prefix code that generates $\operatorname{Im}(f)$ is denoted by $\operatorname{imC}(f)$, and is called the image code. The following inverse monoid is a stepping stone towards defining $G_{k, 1}$ :

$$
\begin{aligned}
& \mathcal{R I}_{A}^{\text {fin }}=\left\{f: f \text { is a right ideal morphism of } A^{*}, f\right. \text { is injective, and } \\
& \operatorname{domC}(f) \text { and } \operatorname{imC}(f) \text { are finite maximal prefix codes\}. }
\end{aligned}
$$

We also write $\mathcal{R} \mathcal{I}_{k}^{\text {fin }}$ for $\mathcal{R} \mathcal{I}_{A}^{\text {fin }}$ (where $k=|A|$ ). It is proved in [1, Prop. 2.1] that every $f \in \mathcal{R} \mathcal{I}_{k}^{\text {fin }}$ is contained in a unique $\subseteq$-maximum right ideal morphism in $\mathcal{R} \mathcal{I}_{k}^{\text {fin }}$; this is called the maximum extension of $f$. The Higman-Thompson group $G_{k, 1}$ (where $k=|A|$ ) is a homomorphic image of $\mathcal{R} \mathcal{I}_{k}^{\text {fin }}$, and also a subset of $\mathcal{R} \mathcal{I}_{k}^{\text {fin }}$ (as a set):

Definition 1.2 (the Higman-Thompson group $G_{k, 1}$ ). The Higman-Thompson group $G_{k, 1}$, as a set, consists of the right ideal morphisms $f \in \mathcal{R I}_{k}^{\text {fin }}$ that are maximum extensions in $\mathcal{R} \mathcal{I}_{k}^{\text {fin }}$. The multiplication in $G_{k, 1}$ consists of composition, followed by maximum extension.

See [1] for a proof that this multiplication turns $G_{k, 1}$ into a group.

Every element $f \in \mathcal{R I}_{k}^{\text {fin }}$ (and in particular, every $f \in G_{k, 1}$ ) is determined by the restriction of $f$ to $\operatorname{domC}(f)$; this is a bijection from the finite prefix code domC $(f)$ onto the finite prefix code $\operatorname{imC}(f)$. We call such a finite bijection a table. We do not assume here that $f$ is a maximum extension, so for an element $f \in G_{k, 1}$ there are many non-maximal tables that determine $f$ by maximal extension. The well-known tree representation of $G_{k, 1}$ is obtained by using the prefix trees of $\operatorname{domC}(f)$ and $\operatorname{imC}(f)$.

Lemma 1.3 The right ideal morphism $f \in \mathcal{R I}_{A}^{\text {fin }}$ determined by a table $F: P \rightarrow Q$ can be extended iff there exist $p, q \in A^{*}$ such that for every $\alpha \in A: \quad p \alpha \in P, q \alpha \in Q$, and $F(p \alpha)=q \alpha$.

In that case, $f$ can be extended by defining $f(p)=q$. So the table for this extension is obtained be replacing $\{(p \alpha, q \alpha): \alpha \in A\}$ by $\{(p, q)\}$ in the table. This is called an extension step of the table $F$.

Proof. See [1, Lemma 2.2].

Since in an extension step the cardinality of $\operatorname{dom} C(f)$ decreases, only finitely steps are needed to reach the maximum extension of $f$.

Notation. For any prefix code $P \subseteq\left\{a_{0}, a_{1}\right\}^{*}$, $\operatorname{spref}(P)$ denotes the set of strict prefixes of the elements of $P$. Formally,

$$
\operatorname{spref}(P)=\left\{x \in\left\{a_{0}, a_{1}\right\}^{*}:(\exists p \in P)\left[x<_{\text {pref }} p\right]\right\} .
$$

\section{Lemma 1.4.}

(1) If $P$ is a finite maximal prefix code over $A_{2}=\left\{a_{0}, a_{1}\right\}$, then $P \cup \operatorname{spref}(P) \cdot\left\{a_{2}, \ldots, a_{k-1}\right\}$ is a finite maximal prefix code over $A_{k}=\left\{a_{0}, a_{1}, \ldots, a_{k-1}\right\}$.

(2) If $P \cup Q \cdot\left\{a_{2}, \ldots, a_{k-1}\right\}$ is a finite maximal prefix code over $A_{k}$, where $P$ and $Q$ are finite subsets of $\left\{a_{0}, a_{1}\right\}^{*}$, then $P$ is a finite maximal prefix code over $\left\{a_{0}, a_{1}\right\}$ and $Q=\operatorname{spref}(P)$.

Proof. See [2, Lemma 9.1], where $k=3$; the general case is similar.

Lemma 1.5 Let $P \subset A^{*}$ be a finite maximal prefix code. Then every $v \in A^{\omega}$ has a unique prefix in P. Formally, $\left(\forall v \in A^{\omega}\right)\left(\exists ! p \in P, u \in A^{\omega}\right)[v=p u]$.

PROOF. The proof is straightforward. 


\section{Embedding $G_{2,1}$ into $G_{k, 1}$}

To embed $G_{2,1}$ into $G_{k, 1}$ the following subgroup of $G_{k, 1}$ is used as an intermediary stage:

$$
\begin{aligned}
G_{k, 1}(0,1|2| \ldots \mid k-1) & \\
=\left\{g \in G_{k, 1}:\right. & (1) \quad \operatorname{domC}(g) \cup \operatorname{imC}(g) \subset\left\{a_{0}, a_{1}\right\}^{*} \cup \bigcup_{i=1}^{k-1}\left\{a_{0}, a_{1}\right\}^{*} a_{i}, \text { and } \\
& (2) \text { for all } x \in A_{k}^{*}: \\
& (2.1) \quad x \in\left\{a_{0}, a_{1}\right\}^{*} \Leftrightarrow g(x) \in\left\{a_{0}, a_{1}\right\}^{*}, \text { and } \\
& \left.(2.2) \quad \text { for all } i=2, \ldots, k-1: x \in\left\{a_{0}, a_{1}\right\}^{*} a_{i} \Leftrightarrow g(x) \in\left\{a_{0}, a_{1}\right\}^{*} a_{i}\right\} .
\end{aligned}
$$

The special case $G_{3,1}(0,1 \mid 2)$ was introduced in [2, Def. 4.4] (in [2] the alphabet $A_{3}=\left\{a_{0}, a_{1}, a_{2}\right\}$ was denoted by $\{0,1, \#\})$.

Lemma 2.1 The group $G_{k, 1}(0,1|2| \ldots \mid k-1)$ consists of the elements of $G_{k, 1}$ with tables of the form

$$
\left[\begin{array}{lll|lll|lll|lll}
u_{1} & \ldots & u_{\ell} & p_{1} a_{2} & \ldots & p_{\ell-1} a_{2} & \ldots & \ldots & \ldots & p_{1} a_{k-1} & \ldots & p_{\ell-1} a_{k-1} \\
v_{1} & \ldots & v_{\ell} & q_{1}^{(2)} a_{2} & \ldots & q_{\ell-1}^{(2)} a_{2} & \ldots & \ldots & \ldots & q_{1}^{(k-1)} a_{k-1} & \ldots & q_{\ell-1}^{(k-1)} a_{k-1}
\end{array}\right]
$$

or equivalently,

$$
\left\{\left(u_{r}, v_{r}\right): 1 \leq r \leq \ell\right\} \cup \bigcup_{i=2}^{k-1}\left\{\left(p_{s} a_{i}, q_{s}^{(i)} a_{i}\right): 1 \leq s \leq \ell-1\right\} .
$$

Here $\left\{u_{r}: 1 \leq r \leq \ell\right\}$ and $\left\{v_{r}: 1 \leq r \leq \ell\right\}$ are maximal prefix codes over $\left\{a_{0}, a_{1}\right\}$ of equal cardinality $\ell \geq 1$, and $\left\{p_{s}: 1 \leq s \leq \ell-1\right\}=\operatorname{spref}\left(\left\{u_{r}: 1 \leq r \leq \ell\right\}\right)$. The map $u_{r} \mapsto v_{r}($ for $1 \leq r \leq \ell)$ is an arbitrary bijection. For every $i=2, \ldots, k-1:\left\{q_{1}^{(i)}, \ldots, q_{\ell-1}^{(i)}\right\}=\operatorname{spref}\left(\left\{v_{1}, \ldots, v_{\ell}\right\}\right)$, and the map $p_{j} a_{i} \mapsto q_{j}^{(i)} a_{i}$ (for $1 \leq j \leq \ell-1$ ) is an arbitrary bijection.

Proof. This follows in a straightforward way from the definition of $G_{k, 1}(0,1|2| \ldots \mid k-1)$ and Lemma 1.4. The fact that for every $a_{i}$, the number of elements $p_{j} a_{i}$ (and $q_{j}^{(i)} a_{i}$ ) is $\ell-1$ follows from the fact that the set of strict prefixes of $\left\{u_{1}, \ldots, u_{\ell}\right\}$ is the set of interior vertices of the prefix tree of $\left\{u_{1}, \ldots, u_{\ell}\right\}$ (over the alphabet $\left\{a_{0}, a_{1}\right\}$ ); see [2, Lemma 4.7].

Definition 2.2 A function $g$ partially fixes a set $S \subseteq A^{*}$ iff $g(x)=x$ for every $x \in S \cap \operatorname{Dom}(g) \cap$ $\operatorname{Im}(g)$. This is also called partial pointwise stabilization. For a subgroup $G \subseteq G_{k, 1}$, the partial fixator (in $G$ ) of $S$ is

$$
\operatorname{pFix}_{G}(S)=\{g \in G:(\forall x \in S \cap \operatorname{Dom}(g) \cap \operatorname{Im}(g))[g(x)=x]\} .
$$

If the set $S$ is a right ideal of $A^{*}$ then $\operatorname{pFix}_{G}(S)$ is a group [2, Lemma 4.1].

\section{Lemma 2.3.}

(1) The group $\mathrm{pFix}_{G_{2,1}}\left(a_{0}\left\{a_{0}, a_{1}\right\}^{*}\right)$ consist of the elements of $G_{2,1}$ that have a table of the form

$$
\left[\begin{array}{llll}
a_{0} & a_{1} u_{1} & \ldots & a_{1} u_{\ell} \\
a_{0} & a_{1} v_{1} & \ldots & a_{1} v_{\ell}
\end{array}\right]
$$

where $\left\{u_{1}, \ldots, u_{\ell}\right\}$ and $\left\{v_{1}, \ldots, v_{\ell}\right\}$ are maximal prefix codes over $\left\{a_{0}, a_{1}\right\}$.

(2) The subgroups $\operatorname{pFix}_{G_{2,1}}\left(a_{1}\left\{a_{0}, a_{1}\right\}^{*}\right)$ and $\operatorname{pFix}_{G_{2,1}}\left(a_{0}\left\{a_{0}, a_{1}\right\}^{*}\right)$ are isomorphic to $G_{2,1}$.

Proof. (1) The form of the tables follows immediately from the definition of $\mathrm{pFix}_{G_{2,1}}\left(a_{0}\left\{a_{0}, a_{1}\right\}^{*}\right)$.

(2) We define an isomorphism $\theta: G_{2,1} \rightarrow \operatorname{Fix}_{G_{2,1}}\left(a_{1}\left\{a_{0}, a_{1}\right\}^{*}\right)$ by

$$
\left[\begin{array}{lll}
x_{1} & \ldots & x_{n} \\
y_{1} & \ldots & y_{n}
\end{array}\right] \longmapsto\left[\begin{array}{llll}
a_{0} & a_{1} x_{1} & \ldots & a_{1} x_{\ell} \\
a_{0} & a_{1} y_{1} & \ldots & a_{1} y_{\ell}
\end{array}\right]
$$

This map is obviously a bijection, and it is easy to check that it is a homomorphism. 
Definition 2.4 (dictionary order). For an alphabet $A_{k}=\left\{a_{0}, a_{1}, \ldots, a_{k-1}\right\}$, totally ordered as $a_{0}<a_{1}<\ldots<a_{k-1}$, the dictionary order on $A_{k}^{*}$ is defined as follows. For any $u, v \in A_{k}^{*}$ :

$u \leq_{\text {dict }} v \quad$ iff

(1) $u \leq_{\text {pref }} v$, or

(2) $u \not_{\text {pref }} v$, and there exist $p, s, t \in A_{k}^{*}$ and $\alpha, \beta \in A_{k}$ such that $u=p \alpha s, v=p \beta$, and $\alpha<\beta$.

In case (2), $p$ is the longest common prefix of $u$ and $v, \alpha$ is the next letter after $p$ in $u$, and $\beta$ is the next letter after $p$ in $v$. Since case (2) rules out case (1), $p$ is strictly shorter than $u$ and $v$, so the letters $\alpha$ and $\beta$ exist, and $\alpha \neq \beta$.

From now on we assume that $A_{k}$ is an ordered alphabet, as in Def. 2.4.

Definition 2.5 (rank function for $\left.\leq_{\text {dict }}\right)$. Let $P \subset A_{k}^{*}$ be a finite set, and let $\left(p_{1}, \ldots, p_{\ell}\right)$ be the list of all the elements of $P$ in increasing dictionary order. Then the rank of $p_{j}$ in $P$ is $\operatorname{rank}_{P}\left(p_{j}\right)=j-1$. Equivalently, $\operatorname{rank}_{P}\left(p_{j}\right)=\left|\left\{q \in P: q<_{\text {dict }} p_{j}\right\}\right|$.

The following concept is crucial for embedding $G_{2,1}$ into $G_{k, 1}(0,1|2| \ldots \mid k-1)$.

Definition 2.6 ( $* a_{i}$-successor). Consider any $a_{i} \in\left\{a_{2}, \ldots, a_{k-1}\right\}$. Let $P \subset\left\{a_{0}, a_{1}\right\}^{*}$ be any finite maximal prefix code with $|P| \geq 2$, and let $\left(p_{1}, \ldots, p_{\ell}\right)$ be the list of all the elements of $P$ in increasing dictionary order on $\left\{a_{0}, a_{1}\right\}^{*}$, where $\ell=|P|$.

For every $p_{j} \in P \backslash\left\{p_{1}\right\}$, the $* a_{i}$-successor $\left(p_{j}\right)_{i}^{\prime}$ of $p_{j}$ is the element of $\operatorname{spref}(P) a_{i}$, defined as follows, assuming $\left(p_{j+1}\right)_{i}^{\prime}, \ldots,\left(p_{\ell}\right)_{i}^{\prime}$ have already been chosen:

$$
\left(p_{j}\right)_{i}^{\prime}=\min \left\{x a_{i} \in \operatorname{spref}(P) a_{i}: p_{j}<_{\text {dict }} x a_{i} \text { and } x a_{i} \notin\left\{\left(p_{j+1}\right)_{i}^{\prime}, \ldots,\left(p_{\ell}\right)_{i}^{\prime}\right\}\right\},
$$

where min uses the dictionary order in $\left\{a_{0}, a_{1}, a_{i}\right\}^{*}$ (i.e., over the three-letter alphabet $\left\{a_{0}, a_{1}, a_{i}\right\}$ ).

In other words, $\left(p_{j}\right)_{i}^{\prime}$ is the nearest right-neighbor of $p_{j}$ in $\operatorname{spref}(P) a_{i}\left(\subset\left\{a_{0}, a_{1}, a_{i}\right\}^{*}\right)$ that has not yet been associated with another $p_{m}$ for $m>j$. In the definition of $p_{\ell},\left\{\left(p_{j+1}\right)_{i}^{\prime}, \ldots,\left(p_{\ell}\right)_{i}^{\prime}\right\}=\varnothing$ (when $j=\ell$ ).

Remarks. For the concept of $* a_{i}$-successor, the three-letter alphabet $\left\{a_{0}, a_{1}, a_{i}\right\}$ (for a chosen $a_{i}$, $2 \leq i<k$ ) must not be confused with the $k$-letter alphabet $A_{k}$ (except, of course, when $k=3$ ).

Note that $\left(p_{1}\right)_{i}^{\prime}$ is not defined. Indeed, if $P$ has $\ell$ elements, $\operatorname{spref}(P)$ has only $\ell-1$ elements; all $p_{j} \in P$ with $j>1$ have a $* a_{i}$-successor, so there is no element left in $\operatorname{spref}(P) a_{i}$ to be the successor of $p_{1}$; this is further clarified by Lemma 2.7, which gives a simple formula for the $* a_{i}$-successor.

Lemma 2.7 (*a $* a_{i}$-successor formula). Let $a_{i} \in\left\{a_{2}, \ldots, a_{k-1}\right\}$, and let $P \subset\left\{a_{0}, a_{1}\right\}^{*}$ be a finite maximal prefix code with $|P| \geq 2$. Then every element of $P \backslash a_{0}^{*}$ can be written (uniquely) in the form $u a_{1} a_{0}^{m}$ (where $u \in\left\{a_{0}, a_{1}\right\}^{*}$ and $\left.m \geq 0\right)$, and its $* a_{i}$-successor is

$$
\left(u a_{1} a_{0}^{m}\right)_{i}^{\prime}=u a_{i} .
$$

The elements of $a_{0}^{*}$ have no $* a_{i}$-successor.

Conversely, for every $u a_{i} \in \operatorname{spref}(P) a_{i}$ we have: $u a_{i}$ is the $* a_{i}$-successor of $u a_{1} a_{0}^{m}$, where $m$ is the unique number such that $u a_{1} a_{0}^{m} \in P \cap u a_{1} a_{0}^{*}$. Different elements of $P \backslash a_{0}{ }^{*}$ have different $* a_{i}$-successors.

Proof. According to the definition of $* a_{i}$-successor, the first element $p_{1} \in P$ has no $* a_{i}$-successor. In every maximal prefix code, $p_{1} \in a_{0}^{*}$, hence elements of $a_{0}^{*}$ have no $* a_{i}$-successor.

Every element of $\left\{a_{0}, a_{1}\right\}^{*} \backslash a_{0}{ }^{*}$ contains an occurrence of $a_{1}$, and hence is of the form $u a_{1} a_{0}^{m}$, for some $u \in\left\{a_{0}, a_{1}\right\}^{*}$ and $m \geq 0$. And $m$ is unique since $P$ is a prefix code.

Obviously, $u a_{1} a_{0}^{m}<_{\text {dict }} u a_{i}$ in the dictionary order determined by $a_{0}<a_{1}<a_{i}$. Let $\left(p_{1}, \ldots, p_{\ell}\right)$ be the list of all the elements of $P$ in increasing dictionary order, where $\ell=|P|$. For $u a_{1} a_{0}^{m} \in$ 
$P \backslash a_{0}^{*}$, let us denote $\operatorname{rank}_{P}\left(u a_{1} a_{0}^{m}\right)$ by $r-1$, so $u a_{1} a_{0}^{m}=p_{r}$. We want to show that if there exists $v \in \operatorname{spref}(P)$ such that $u a_{1} a_{0}^{m}<_{\text {dict }} v a_{i}<_{\text {dict }} u a_{i}$, then $v a_{i} \in\left\{\left(p_{r+1}\right)_{i}^{\prime}, \ldots,\left(p_{\ell}\right)_{i}^{\prime}\right\}$. This will imply that $u a_{i}$ is the minimum element in $\operatorname{spref}(P) \backslash\left\{\left(p_{r+1}\right)_{i}^{\prime}, \ldots,\left(p_{\ell}\right)_{i}^{\prime}\right\}$, satisfying $p_{r}<_{\text {dict }} u a_{i}$; hence, $u a_{i}=\left(p_{r}\right)_{i}^{\prime}$ by Def. 2.6.

The relations $v \in\left\{a_{0}, a_{1}\right\}^{*}$ and $u a_{1} a_{0}^{m}<_{\text {dict }} v a_{i}<_{\text {dict }} u a_{i}$ imply that $v=u a_{1} x$ for some $x \in$ $\left\{a_{0}, a_{1}\right\}^{*}$.

If $m=0$ then $p_{r}=u a_{1}$ is a prefix of $v=u a_{i} x$, which contradicts the fact that $v \in \operatorname{spref}(P)$. Hence, $\left(u a_{1}\right)_{i}^{\prime}=u a_{i}$. In particular, the case $m=0$ applies to $p_{\ell}$, since in a maximal prefix code the last element belongs to $a_{1}^{*}$, i.e., $p_{\ell}=a_{1}^{n}$ for some $n>0$; hence $\left(p_{\ell}\right)_{i}^{\prime}=a_{1}^{n-1} a_{i}$; so the $* a_{i}$-successor formula holds.

Let us now assume by induction on decreasing $r$ (ranging from $\ell$ down to 2 ), that for all $j>r$ :

if there exists $v_{j} \in \operatorname{spref}(P)$ such that $p_{j}=u_{j} a_{1} a_{0}^{m_{j}}<_{\text {dict }} v_{j} a_{i}<_{\text {dict }} u_{j} a_{i}$, then $v_{j} a_{i} \in\left\{\left(p_{j+1}\right)_{i}^{\prime}, \ldots,\left(p_{\ell}\right)_{i}^{\prime}\right\}$.

The inductive assumption holds when $r=\ell$ (since $v_{\ell}$ does not exist, by the case $m=0$ ). So we can assume that $m>0$, since we already proved that $\left(u a_{1}\right)^{\prime}=u a_{i}$. We want to prove the inductive hypothesis for $r$, i.e.: If there exists $v_{r} \in \operatorname{spref}(P)$ such that $p_{r}=u a_{1} a_{0}^{m}<_{\text {dict }} v_{r} a_{i}<_{\text {dict }} u a_{i}$, then $v_{r} a_{i} \in\left\{\left(p_{r+1}\right)_{i}^{\prime}, \ldots,\left(p_{\ell}\right)_{i}^{\prime}\right\}$.

Case where $u a_{1} a_{0}^{m}<_{\text {dict }} v_{r}$ :

Then $p_{r}=u a_{1} a_{0}^{m}<_{\text {dict }} v_{r}<_{\text {dict }} v_{r} z<_{\text {dict }} v_{r} a_{i}<_{\text {dict }} u a_{i}$ for every $z \in\left\{a_{0}, a_{1}\right\}^{+}$. Since $P$ is maximal, $v_{r}$ is the prefix of some $p_{j}=v_{r} z_{j} \in P$, hence $p_{r}=u a_{1} a_{0}^{m}<_{\text {dict }} p_{j}<_{\text {dict }} v_{r} a_{i}<_{\text {dict }} u a_{i}$, hence $j>r$. Now by induction (since $j>r), v_{r} a_{i} \in\left\{\left(p_{j}\right)_{i}^{\prime}, \ldots,\left(p_{\ell}\right)_{i}^{\prime}\right\} \quad\left(\subseteq\left\{\left(p_{r+1}\right)_{i}^{\prime}, \ldots,\left(p_{\ell}\right)_{i}^{\prime}\right\}\right)$.

Case where $v_{r}=u a_{1} x \leq_{\text {dict }} u a_{1} a_{0}^{m}$ :

Then $v_{r}=u a_{1} a_{0}^{k}$ for some $k<m$ (we cannot have $k=m$, since $v_{r} \in \operatorname{spref}(P)$, i.e., $v_{r}$ is a strict prefix). In that case, $p_{r}=u a_{1} a_{0}^{m}<_{\text {dict }} v_{r} a_{1}=u a_{1} a_{0}^{k} a_{1} \leq_{\text {dict }} u a_{1} a_{0}^{k} a_{1} z<_{\text {dict }} v_{r} a_{i}<_{\text {dict }}$ $u a_{i}$ for all $z \in\left\{a_{0}, a_{1}\right\}^{*}$. Since $P$ is maximal, $v_{r} a_{1}$ is a prefix of some $p_{j}=v_{r} a_{1} z_{j} \in P$, hence $p_{r}<_{\text {dict }} v_{r} a_{1} \leq_{\text {dict }} p_{j}<_{\text {dict }} v_{r} a_{i}<_{\text {dict }} u a_{i}$, hence $j>r$. Then by induction, $v_{r} a_{i} \in\left\{\left(p_{j}\right)_{i}^{\prime}, \ldots,\left(p_{\ell}\right)_{i}^{\prime}\right\}$ $\left(\subseteq\left\{\left(p_{r+1}\right)_{i}^{\prime}, \ldots,\left(p_{\ell}\right)_{i}^{\prime}\right\}\right)$.

In conclusion, if there exists $v_{r} \in \operatorname{spref}(P)$ such that $p_{r}=u a_{1} a_{0}^{m}<_{\text {dict }} v_{r} a_{i}<_{\text {dict }} u a_{i}$, then $v_{r} a_{i} \in\left\{\left(p_{r+1}\right)_{i}^{\prime}, \ldots,\left(p_{\ell}\right)_{i}^{\prime}\right\}$. So, $\left(p_{r}\right)_{i}^{\prime}=u a_{i}$.

For the converse, we saw in Lemma 1.5 that if $P$ is a finite maximal prefix code then every $w \in\left\{a_{0}, a_{1}\right\}^{\omega}$ has a unique prefix in $P$. By taking $w=u a_{1} a_{0}^{\omega}$ we conclude that $P \cap u a_{1} a_{0}^{*}$ is a singleton; i.e., $u$ determines $m$. Since $u$ determines $m$, it follows that the function $u a_{1} a_{0}^{m} \in P \backslash a_{0}^{*} \longmapsto u a_{i}$ is injective. The converse follows immediately now from the fact that $\left(u a_{1} a_{0}^{m}\right)_{i}^{\prime}=u a_{i}$.

Lemma 2.8 Let $a_{i} \in\left\{a_{2}, \ldots, a_{k-1}\right\}$, and let $P \subset\left\{a_{0}, a_{1}\right\}^{*}$ be a finite maximal prefix code, ordered as $p_{1}<_{\text {dict }} \ldots<_{\text {dict }} p_{\ell}$, where $\ell=|P| \geq 2$. Then

(1) $P \cup \operatorname{spref}(P) a_{i}$ is a finite maximal prefix code over the three-letter alphabet $\left\{a_{0}, a_{1}, a_{i}\right\}$.

Moreover, $P \cup \operatorname{spref}(P)\left\{a_{2}, \ldots, a_{k-1}\right\}$ is a finite maximal prefix code over $A_{k}$.

(2) $\left\{\left(p_{2}\right)_{i}^{\prime}, \ldots,\left(p_{\ell}\right)_{i}^{\prime}\right\}=\operatorname{spref}(P) a_{i} \quad\left(\subset\left\{a_{0}, a_{1}, a_{i}\right\}^{*}\right)$.

(3) Consider a one-step restriction, in which $P$ is replaced by $P_{r}=\left(P \backslash\left\{p_{r}\right\}\right) \cup p_{r}\left\{a_{0}, a_{1}\right\}$. Then $\left(p_{r} a_{0}\right)_{i}^{\prime}$ and $\left(p_{r} a_{1}\right)_{i}^{\prime}$ are uniquely determined by $p_{r}$ as follows:

- if $2 \leq r \leq \ell$ then $\left(p_{r} a_{1}\right)_{i}^{\prime}=p_{r} a_{i}$ and $\left(p_{r} a_{0}\right)_{i}^{\prime}=\left(p_{r}\right)_{i}^{\prime}$;

- if $r=1$ then $p_{1} \in P \cap a_{0}^{*}$, hence $p_{1} a_{0} \in P_{1} \cap a_{0}^{*}$, and $p_{1} a_{1} \in P_{1} \backslash a_{0}^{*}$; so $\left(p_{1}\right)_{i}^{\prime}$ and $\left(p_{1} a_{0}\right)_{i}^{\prime}$ do not exist; but $\left(p_{1} a_{1}\right)_{i}^{\prime}=p_{1} a_{i}$ exists.

Proof. (1) is straightforward. (2) follows immediately from Lemma 2.7 and the fact that for any finite prefix code $P$ over $\left\{a_{0}, a_{1}\right\},|\operatorname{spref}(P)|=|P|-1$. 
(3) Let $p_{r}=u a_{1} a_{0}^{m} \in P \backslash a_{0}^{m}$, so $\left(p_{r}\right)_{i}^{\prime}=u a_{i}$ (by Lemma 2.7). By Lemma 1.3, $P_{r}$ is a maximal prefix code over $\left\{a_{0}, a_{1}\right\}$. Applying Lemma 2.7 to $P_{r}$ and its elements $p_{r} a_{0}=u a_{1} a_{0}^{m+1}$ and $p_{r} a_{1}=$ $u a_{1} a_{0}^{m} a_{1}$, we obtain $\left(p_{r} a_{0}\right)^{\prime}=u a_{i}=\left(p_{r}\right)_{i}^{\prime}$ and $\left(p_{r} a_{1}\right)_{i}^{\prime}=u a_{1} a_{0}^{m} a_{i}=p_{r} a_{i}$.

If $p_{1}=a_{0}^{m} \in P \cap a_{0}^{*}$ (for some $m \geq 0$ ), then $p_{1} a_{0}=a_{0}^{m+1}$, and $p_{1} a_{1}=a_{0}^{m} a_{1}$. Hence $\left(p_{1}\right)_{i}^{\prime}$ and $\left(p_{1} a_{0}\right)_{i}^{\prime}$ do not exist. But $\left(p_{1} a_{1}\right)_{i}^{\prime}=a_{0}^{m} a_{i}=p_{1} a_{i}$.

Lemma 2.9 For every $k \geq 3$ there exists an embedding $\iota: G_{2,1} \hookrightarrow G_{k, 1}(0,1|2| \ldots \mid k-1)$.

Proof. We define the embedding

$$
\begin{aligned}
& g=\left[\begin{array}{lll}
p_{1} & \cdots & p_{\ell} \\
q_{1} & \ldots & q_{\ell}
\end{array}\right] \stackrel{\iota}{\hookrightarrow}
\end{aligned}
$$

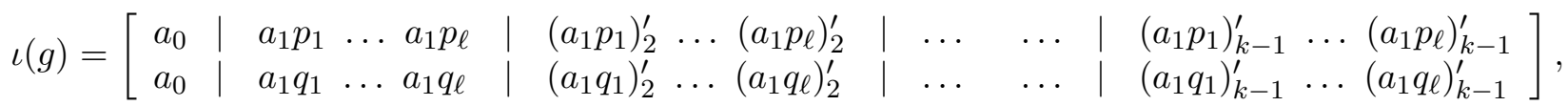

where $\left\{p_{1}, \ldots, p_{\ell}\right\}$ and $\left\{q_{1}, \ldots, q_{\ell}\right\}$ are finite maximal prefix codes over $\left\{a_{0}, a_{1}\right\}$. Equivalently, the table for $\iota(g)$ is

$$
\left\{\left(a_{0}, a_{0}\right)\right\} \cup\left\{\left(a_{1} p_{r}, a_{1} q_{r}\right): 1 \leq r \leq \ell\right\} \cup \bigcup_{i=2}^{k-1}\left\{\left(\left(a_{1} p_{r}\right)_{i}^{\prime},\left(a_{1} q_{r}\right)_{i}^{\prime}\right): 1 \leq r \leq \ell\right\} .
$$

The function $\iota$ is well defined, as a function between tables. Indeed, for all $r=1, \ldots, \ell:\left(a_{1} p_{r}\right)^{\prime}$ determines $p_{r}$ (by Lemma 2.7), which in turn determines $q_{r}$ (via the table for $g$ ), which determines $\left(a_{1} q_{r}\right)^{\prime}$. And $\iota$ is obviously injective.

To show that $\iota$ is also a map from $G_{2,1}$ to $G_{k, 1}(0,1|2| \ldots \mid k-1)$, we show that the operation of one-step restriction commutes with $\iota$. Moreover, after that we can restrict tables so that when we compose two tables, the image row of the first table is equal to the domain row of the second; this makes it easy to show that $\iota$ is a homomorphism.

For any $g \in G_{2,1}$, given by a table $\left\{\left(p_{r}, q_{r}\right): 1 \leq r \leq \ell\right\}$, the restriction of $g$ at $p_{s}$ (for $\left.1 \leq s \leq \ell\right)$ is given by the table

$$
\operatorname{restr}_{p_{s}}(g)=\left\{\left(p_{s} a_{0}, q_{s} a_{0}\right),\left(p_{s} a_{1}, q_{s} a_{1}\right)\right\} \cup\left\{\left(p_{j}, q_{j}\right): 1 \leq j \leq \ell, j \neq s\right\} .
$$

Similarly, for any $f \in G_{k, 1}$, given by a table $\left\{\left(u_{j}, v_{j}\right): 1 \leq j \leq m\right\}$, the restriction at $u_{t}($ for $1 \leq t \leq m)$ is given by the table

$$
\operatorname{restr}_{u_{t}}(f)=\left\{\left(u_{t} a_{i}, v_{t} a_{i}\right): a_{i} \in A_{k}\right\} \cup\left\{\left(u_{j}, v_{j}\right): 1 \leq j \leq m, j \neq t\right\} .
$$

(1) (Commutation:) Verification that $\iota\left(\operatorname{restr}_{p_{r}}(g)\right)=\operatorname{restr}_{a_{1} p_{r}}(\iota(g)) \quad($ for $r=1, \ldots, \ell)$ :

$$
\begin{aligned}
& \operatorname{restr}_{p_{r}}(g)=\left[\begin{array}{lllllll}
\ldots & p_{r-1} & p_{r} a_{0} & p_{r} a_{1} & p_{r+1} & \ldots \\
\ldots & q_{r-1} & q_{r} a_{0} & q_{r} a_{1} & q_{r+1} & \ldots
\end{array}\right] \stackrel{\iota}{\hookrightarrow} \quad \iota\left(\operatorname{restr}_{p_{r}}(g)\right) \\
& =\left[\begin{array}{lllllllllllll}
\ldots & a_{1} p_{r-1} & a_{1} p_{r} a_{0} & a_{1} p_{r} a_{1} & a_{1} p_{r+1} & \ldots & \ldots & \left(a_{1} p_{r-1}\right)_{i}^{\prime} & \left(a_{1} p_{r} a_{0}\right)_{i}^{\prime} & \left(a_{1} p_{r} a_{1}\right)_{i}^{\prime} & \left(a_{1} p_{r+1}\right)_{i}^{\prime} & \ldots \\
\ldots & a_{1} q_{r-1} & a_{1} q_{r} a_{0} & a_{1} q_{r} a_{1} & a_{1} q_{r+1} & \ldots & \ldots & \left(a_{1} q_{r-1}\right)_{i}^{\prime} & \left(a_{1} q_{r} a_{0}\right)_{i}^{\prime} & \left(a_{1} q_{r} a_{1}\right)_{i}^{\prime} & \left(a_{1} q_{r+1}\right)_{i}^{\prime} & \ldots
\end{array}\right] \\
& =\left[\begin{array}{llllllllllll}
\ldots & a_{1} p_{r-1} & a_{1} p_{r} a_{0} & a_{1} p_{r} a_{1} & a_{1} p_{r+1} & \ldots & \ldots & \left(a_{1} p_{r-1}\right)_{i}^{\prime} & \left(a_{1} p_{r}\right)_{i}^{\prime} & a_{1} p_{r} a_{i} & \left(a_{1} p_{r+1}\right)_{i}^{\prime} & \ldots \\
\ldots & a_{1} q_{r-1} & a_{1} q_{r} a_{0} & a_{1} q_{r} a_{1} & a_{1} q_{r+1} & \ldots & \ldots & \left(a_{1} q_{r-1}\right)_{i}^{\prime} & \left(a_{1} q_{r}\right)_{i}^{\prime} & a_{1} q_{r} a_{i} & \left(a_{1} q_{r+1}\right)_{i}^{\prime} & \ldots
\end{array}\right] ;
\end{aligned}
$$

the latter equality holds by Lemma 2.8. Here, $a_{i}$ ranges over $\left\{a_{2}, \ldots, a_{k-1}\right\}$. Recall that here the restriction restr $p_{r}$ happens over the alphabet $\left\{a_{0}, a_{1}\right\}$.

Equivalently, the latter table for $\iota\left(\operatorname{restr}_{p_{r}}(g)\right)$ is

$$
\begin{aligned}
& \left\{\left(a_{0}, a_{0}\right)\right\} \cup\left\{\left(a_{1} p_{r} a_{0}, a_{1} q_{r} a_{0}\right),\left(a_{1} p_{r} a_{1}, a_{1} q_{r} a_{1}\right)\right\} \quad \cup \quad\left\{\left(a_{1} p_{j}, a_{1} q_{j}\right): 1 \leq j \leq \ell, j \neq r\right\} \\
& \cup \bigcup_{i=2}^{k-1}\left(\left\{\left(\left(a_{1} p_{r}\right)_{i}^{\prime},\left(a_{1} q_{r}\right)_{i}^{\prime}\right),\left(a_{1} p_{r} a_{i}, a_{1} q_{r} a_{i}\right)\right\} \cup\left\{\left(\left(a_{1} p_{j}\right)_{i}^{\prime},\left(a_{1} q_{j}\right)_{i}^{\prime}\right): 1 \leq j \leq \ell, j \neq r\right\}\right) .
\end{aligned}
$$


On the other hand, the table for $\operatorname{restr}_{a_{1} p_{r}}(\iota(g))$ in $G_{k, 1}$ is

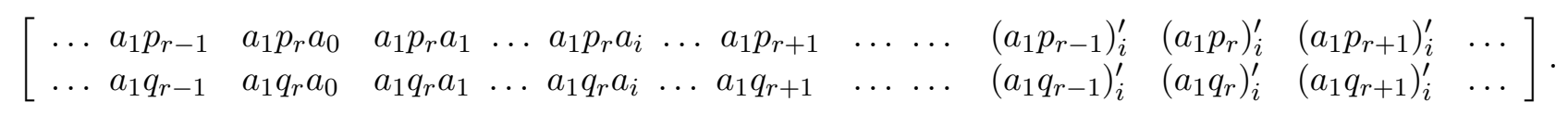

Recall that the restriction $\operatorname{restr}_{a_{1} p_{r}}$ happens over the alphabet $A_{k}$.

Equivalently, the latter table for $\operatorname{restr}_{a_{1} p_{r}}(\iota(g))$ is

$$
\begin{aligned}
& \left\{\left(a_{0}, a_{0}\right)\right\} \\
& \cup \quad\left\{\left(a_{1} p_{r} a_{0}, a_{1} q_{r} a_{0}\right), \ldots,\left(a_{1} p_{r} a_{i}, a_{1} q_{r} a_{i}\right), \ldots,\left(a_{1} p_{r} a_{k-1}, a_{1} q_{r} a_{k-1}\right)\right\} \\
& \cup \quad\left\{\left(a_{1} p_{j}, a_{1} q_{j}\right): 1 \leq j \leq \ell, j \neq r\right\} \\
& \cup \quad \bigcup_{i=2}^{k-1}\left\{\left(\left(a_{1} p_{j}\right)_{i}^{\prime},\left(a_{1} q_{j}\right)_{i}^{\prime}\right): 1 \leq j \leq \ell\right\} .
\end{aligned}
$$

We see that the tables for $\iota\left(\operatorname{restr}_{p_{r}}(g)\right)$ and $\operatorname{restr}_{a_{1} p_{r}}(\iota(g))$ are the same, up to the order of the entries. More precisely,

$$
\begin{aligned}
\iota\left(\operatorname{restr}_{p_{r}}(g)\right) & =\left\{\left(a_{0}, a_{0}\right)\right\} \cup\left\{\left(a_{1} p_{r} a_{0}, a_{1} q_{r} a_{0}\right),\left(a_{1} p_{r} a_{1}, a_{1} q_{r} a_{1}\right)\right\} \quad \cup \quad\left\{\left(a_{1} p_{j}, a_{1} q_{j}\right): 1 \leq j \leq \ell, j \neq r\right\} \\
& \cup \bigcup_{i=2}^{k-1}\left(\left\{\left(\left(a_{1} p_{r}\right)_{i}^{\prime},\left(a_{1} q_{r}\right)_{i}^{\prime}\right),\left(a_{1} p_{r} a_{i}, a_{1} q_{r} a_{i}\right)\right\} \cup\left\{\left(\left(a_{1} p_{j}\right)_{i}^{\prime},\left(a_{1} q_{j}\right)_{i}^{\prime}\right): 1 \leq j \leq \ell, j \neq r\right\}\right) . \\
= & \left.\left\{\left(a_{0}, a_{0}\right)\right\} \cup\left\{\left(a_{1} p_{r} a_{0}, a_{1} q_{r} a_{0}\right),\left(a_{1} p_{r} a_{1}, a_{1} q_{r} a_{1}\right)\right\} \cup \quad \cup\left(a_{1} p_{j}, a_{1} q_{j}\right): 1 \leq j \leq \ell, j \neq r\right\} \\
& \cup\left\{\left(a_{1} p_{r} a_{i}, a_{1} q_{r} a_{i}\right): 2 \leq i \leq k-1\right\} \\
& \cup \bigcup_{i=2}^{k-1}\left(\left\{\left(\left(a_{1} p_{r}\right)_{i}^{\prime},\left(a_{1} q_{r}\right)_{i}^{\prime}\right)\right\} \cup\left\{\left(\left(a_{1} p_{j}\right)_{i}^{\prime},\left(a_{1} q_{j}\right)_{i}^{\prime}\right): 1 \leq j \leq \ell, j \neq r\right\}\right) . \\
= & \left\{\left(a_{0}, a_{0}\right)\right\} \cup\left\{\left(a_{1} p_{r} a_{0}, a_{1} q_{r} a_{0}\right), \ldots,\left(a_{1} p_{r} a_{i}, a_{1} q_{r} a_{i}\right), \ldots,\left(a_{1} p_{r} a_{k-1}, a_{1} q_{r} a_{k-1}\right)\right\} \\
& \cup\left\{\left(a_{1} p_{j}, a_{1} q_{j}\right): 1 \leq j \leq \ell, j \neq r\right\} \\
& \cup \bigcup_{i=2}^{k-1}\left\{\left(\left(a_{1} p_{j}\right)_{i}^{\prime},\left(a_{1} q_{j}\right)_{i}^{\prime}\right): 1 \leq j \leq \ell\right\} \\
= & \operatorname{restr}_{a_{1} p_{r}}(\iota(g)) .
\end{aligned}
$$

(2) To complete the proof that $\iota$ is a homomorphism, consider $h, g \in G_{2,1}$ with tables

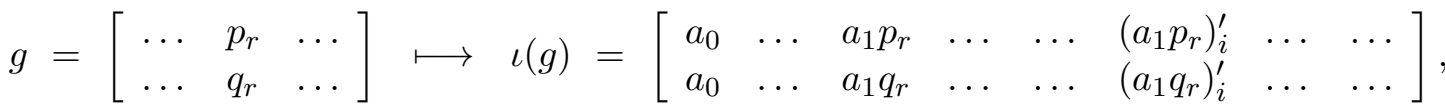

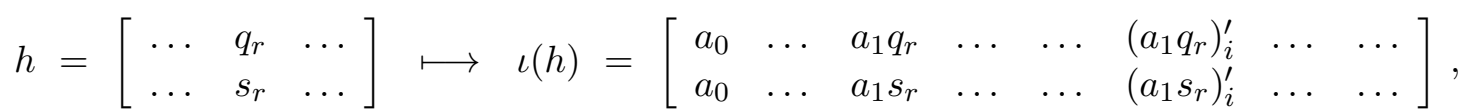

where $r=1, \ldots, \ell$.

We can indeed assume that the output row of $\iota(g)$ is equal to the input row of $\iota(h)$, since we proved that one-step restrictions commute with $\iota$. We just need the obtain row equality on the $\left\{a_{0}, a_{1}\right\}^{*}$-part of the table; the remainder of the rows are then equal too, since $q_{r}$ determines $\left(a_{1} q_{r}\right)_{i}^{\prime}$.

Then by composing the tables we obtain

$$
\iota(h) \circ \iota(g)=\left[\begin{array}{llllllll}
a_{0} & \ldots & a_{1} p_{r} & \ldots & \ldots & \left(a_{1} p_{r}\right)_{i}^{\prime} & \ldots & \ldots \\
a_{0} & \ldots & a_{1} s_{r} & \ldots & \ldots & \left(a_{1} s_{r}\right)_{i}^{\prime} & \ldots & \ldots
\end{array}\right],
$$

while $h \circ g$ has a table

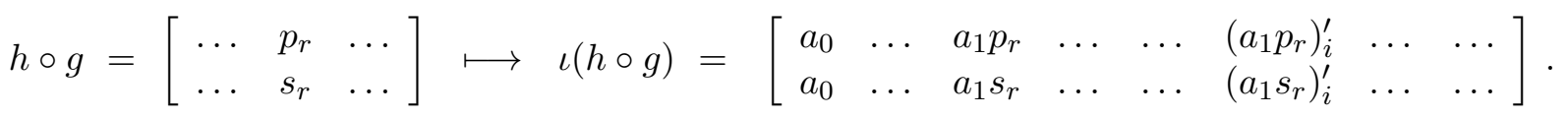

So, $\iota(h) \circ \iota(g)=\iota(h \circ g)$.

This completes the proof of Theorem 1.1. 


\section{Comments}

(1) Explanation of Higman's numbers $K=1+(k-1) d$ for $d \geq 1$ :

For $K>k$, Higman's embedding $G_{K, 1} \leq G_{k, 1}$ uses a bijective encoding of the alphabet $A_{K}=$ $\left\{a_{0}, a_{1}, \ldots, a_{K-1}\right\}$ onto any maximal prefix code of size $K$ over $A_{k}$. Any maximal prefix code over the alphabet $A_{k}$ has cardinality $1+(k-1) d$ for some $d \geq 1$, where $d$ is the number of interior vertices in the prefix tree of the maximal prefix code. This gives the possible values of $K$ for this method.

(2) For the embedding $G_{2,1} \leq G_{k, 1}$ with $k>2$, the above encoding method does not work; obviously, there is no maximal prefix code of size 2 over $A_{k}$.

In [3], $F_{2,1}$ is embedded into $F_{k, 1}$ by ignoring the middle edges in every caret (just keeping the edges labeled by $a_{0}$ and $a_{k-1}$ ), where $a_{k-1}$ now represents the letter $a_{1}$ used by $F_{2,1}$. This works because (by the dictionary order preserving property of $F_{k, 1}$ ), the mapping of the end edges of a caret determines the mapping of the intermediary edges (see [3]). For $G_{k, 1}$ with $k>2$, this does not work: the mapping of the intermediary edges of a caret is not determined by the mapping of the end edges; for $G_{2,1}$ versus $G_{k, 1}$, this "embedding" is not a function.

Higman's embeddings, and the embedding $F_{h, 1}<F_{k, 1}$ for all $h, k \geq 2$, seem to be the basis of the general belief that all $G_{k, 1}$ easily embed in one another. In Matte Bon's paper [6], the general embedding result is actually not stated explicitly, although it follows immediately from [6. Cor. 11.16]; however, in [6] it is stated more than once that these embeddings are "well known".

(3) The idea for constructing an embedding $G_{2,1} \leq G_{k, 1}$ with $k>2$ can be developed through a sequence of ideas that do not quite work. We illustrate this with $k=3$, since this captures most of the difficulty. (This came about independently of Matte Bon's proof, which I did not know of.)

Consider any $G_{2,1}$-table $t=\left\{\left(p_{j}, q_{j}\right): 1 \leq j \leq \ell\right\}$, where $P=\left\{p_{j}: 1 \leq j \leq \ell\right\}$ and $Q=\left\{q_{j}: 1 \leq\right.$ $j \leq \ell\}$ are finite maximal prefix codes over $A_{2}=\{0,1\}$.

- First idea: Map the $G_{2,1}$-table $t$ to the table $\left\{\left(p_{j}, q_{j}\right): 1 \leq j \leq \ell\right\} \cup\{(2,2)\}$. This obviously does not work, because $P \cup\{2\}$ is not a maximal prefix code over $A_{3}=\{0,1,2\}$ (except if $P=\{0,1\}$ ).

- Second idea: Use the fact that $P \cup \operatorname{spref}(P) 2$ is a maximal prefix code over $A_{3}$ (Lemma 1.4).

Problem: How should spref $(P) 2$ be mapped bijectively onto $\operatorname{spref}(Q) 2$ ? The simple matching by dictionary order yields an injective map of $G_{2,1}$-tables to $G_{3,1}$-tables; but this map between tables is not a map from $G_{2,1}$ into $G_{3,1}$ (since the table map does not commute with restriction - see the proof of Lemma 2.9).

- Third idea: Introduce the concept of $* 2$-successor, and map $t=\left\{\left(p_{j}, q_{j}\right): 1 \leq j \leq \ell\right\}$ to $\left\{\left(p_{j}, q_{j}\right): 1 \leq j \leq \ell\right\} \cup\left\{\left(\left(p_{j}\right)^{\prime},\left(q_{j}\right)^{\prime}\right): 2 \leq j \leq \ell\right\}$.

Problem: Elements of $0^{*}$ have no $* 2$-successor; we let $p_{1} \in 0^{*}$, which solves the problem in $P$ (we assume here that $P$ is written in increasing dictionary order; but $Q$ isn't). But the $0^{*}$ element in $Q$ might not be $q_{1}$. So we still don't know how to map all the $* 2$-successors.

- Fourth idea: Assume $P$ and $Q$ are ordered by the dictionary order of $A_{2}^{*}$ as $p_{1}<_{\text {dict }} \ldots<_{\text {dict }} p_{\ell}$, and $q_{1}<_{\text {dict }} \ldots<_{\text {dict }} q_{\ell}$. We map the table $t$ to the $G_{3,1}(0,1 ; 2)$-table

$$
\left[\begin{array}{llllll}
p_{1} & \ldots & p_{\ell} & \left(p_{2}\right)^{\prime} & \ldots & \left(p_{\ell}\right)^{\prime} \\
q_{\pi(1)} & \ldots & q_{\pi(\ell)} & \left(q_{\bar{\pi}(2)}\right)^{\prime} & \ldots & \left(q_{\bar{\pi}(\ell)}\right)^{\prime}
\end{array}\right],
$$

where $\pi$ is a permutation of $\{1,2, \ldots, \ell\}$, and $\bar{\pi}$ is the permutation of $\{2, \ldots, \ell\}$ determined by $\pi$ as follows. For $i \in\{2, \ldots, \ell\}$,

$$
\bar{\pi}(i)= \begin{cases}\pi(i) & \text { if } \pi(i) \in\{2, \ldots, \ell\}, \\ \pi(1) & \text { otherwise (i.e., if } \pi(i)=1) .\end{cases}
$$

This is an injection of $G_{2,1}$ into $G_{k, 1}$; but it is not a homomorphism because we do not have $\overline{\rho \circ \pi}=\bar{\rho} \circ \bar{\pi}$ in general. A counter-example is

$$
\pi=\left[\begin{array}{llll}
1 & 2 & 3 & 4 \\
2 & 4 & 1 & 3
\end{array}\right], \quad \rho=\left[\begin{array}{llll}
1 & 2 & 3 & 4 \\
3 & 4 & 1 & 2
\end{array}\right]
$$


- Finally: To the idea of the $* 2$-successor, add the replacement of $G_{2,1}$ by its isomorphic copy $\operatorname{pFix}_{G_{2,1}}\left(0\{0,1\}^{*}\right)$; this is carried out in section 2 .

(4) The asymmetry between the embeddings $G_{k, 1} \leq G_{2,1}$ and $G_{2,1} \leq G_{k, 1}$ is interesting. Matte Bon's [6. Cor. 11.18] implies that every copy of $G_{2,1}$ in $G_{k, 1}$ (both acting on $A_{k}^{\omega}$ ) has a non-empty clopen set of global fixed points (in our Lemma 2.9, this is $a_{0} A_{k}^{\omega}$ ), while such fixed points do not generally exist for $G_{k, 1} \leq G_{2,1}$.

Intuitively, the difficulty about embedding $G_{2,1}$ into $G_{k, 1}$ comes from the fact that a $G_{k, 1}$-table contains more entries than a $G_{2,1}$-table; how can a $G_{2,1}$-table determine this extra entries? For $F_{2,1} \leq F_{k, 1}$, order-preservation makes that determination [3]. For $G_{k, 1} \leq G_{2,1}$ the problem is reversed, and coding solves the problem [5]. For $G_{2,1} \leq G_{k, 1}$, the concept of $* a_{i}$-successor is the main idea for making a $G_{2,1}$-table determine a $G_{k, 1}$-table; in addition, in order to get a homomorphism, the idea of replacing $G_{2,1}$ by its isomorphic copy $\operatorname{pFix}_{G_{2,1}}\left(a_{0}\left\{a_{0}, a_{1}\right\}^{*}\right)$ plays a crucial role. This makes sense in view of [6, Cor. 11.18] (which I did not know when version 1 of this was written).

Acknowledgements: I would like to thank Matt Brin for many discussions, in particular for pointing out the idea for the mutual embeddings of the groups $F_{k, 1}$.

I'm grateful to Nicolás Matte Bon for contacting me after I posted the first version of this paper (arXiv.org/abs/1902.09414v1).

\section{References}

[1] J.C. Birget, "The groups of Richard Thompson and complexity", International J. of Algebra and Computation 14(5,6) (Dec. 2004) 569-626. Preprint: https://arxiv.org/abs/math/0204292

[2] J.C. Birget, "Circuits, coNP-completeness, and the groups of Richard Thompson", International J. of Algebra and Computation 16(1) (Feb. 2006) 35-90. Preprint: https://arxiv.org/abs/math/0310335

[3] J. Burillo, S. Cleary, and M.I. Stein, " Metrics and embeddings of generalizations of Thompson's group F". Trans. Amer. Math. Soc. 353(4) (2001) 16771689.

[4] J.W. Cannon, W.J. Floyd, W.R. Parry, "Introductory notes on Richard Thompson's groups", L'Enseignement Mathématique 42 (1996) 215-256.

[5] G. Higman, "Finitely presented infinite simple groups", Notes on Pure Mathematics 8, The Australian National University, Canberra (1974).

[6] N. Matte Bon, "Rigidity properties of full groups of pseudogroups over the Cantor set". https://arxiv.org/pdf/1801.10133.pdf (10 Dec. 2018)

[7] R. McKenzie, R.J. Thompson, "An elementary construction of unsolvable word problems in group theory", in Word Problems, (W.W. Boone, F.B. Cannonito, R.C. Lyndon, editors), North-Holland (1973) pp. 457478.

[8] E. Pardo, "The isomorphism problem for Higman-Thompson groups", J. Algebra 344 (2011) 172-183.

[9] E.A. Scott, "A construction which can be used to produce finitely presented infinite simple groups", J. of Algebra 90 (1984) 294-322.

[10] Richard J. Thompson, Manuscript (1960s).

[11] R.J. Thompson, "Embeddings into finitely generated simple groups which preserve the word problem", in Word Problems II, (S. Adian, W. Boone, G. Higman, editors), North-Holland (1980) pp. 401-441.

Dept. of Computer Science, and CCIB

Rutgers University - Camden

birget@camden.rutgers.edu 